\title{
Sulforaphane exerts anti-inflammatory effects against lipopolysaccharide-induced acute lung injury in mice through the Nrf2/ARE pathway
}

\author{
TIANJIE QI ${ }^{1}$, FEI XU ${ }^{2}$, XIXIN YAN $^{1}$, SHUAI LI $^{1}$ and HAITAO LI ${ }^{1}$ \\ ${ }^{1}$ Department of Respiratory Medicine, The Second Hospital of Hebei Medical University, Shijiazhuang, Hebei 050000; \\ ${ }^{2}$ Department of Cardiology, Jin Zhou People's Hospital of Hebei Province, Hebei 052260, P.R. China
}

Received May 6, 2015; Accepted October 16, 2015

DOI: $10.3892 / \mathrm{ijmm} .2015 .2396$

\begin{abstract}
Sulforaphane (1-isothiocyanate-4-methyl sulfonyl butane) is a plant extract (obtained from cruciferous vegetables, such as broccoli and cabbage) and is known to exert anticancer, antioxidant and anti-inflammatory effects. It stimulates the generation of human or animal cells, which is beneficial to the body. The aim of the current study was to determine whether sulforaphane protects against lipopolysaccharide (LPS)-induced acute lung injury (ALI) through its anti-inflammatory effects, and to investigate the signaling pathways involved. For this purpose, male BALB/c mice were treated with sulforaphane $(50 \mathrm{mg} /$ $\mathrm{kg}$ ) and 3 days later, ALI was induced by the administration of LPS $(5 \mathrm{mg} / \mathrm{kg})$ and we thus established the model of ALI. Our results revealed that sulforaphane significantly decreased lactate dehydrogenase (LDH) activity (as shown by LDH assay), the wet-to-dry ratio of the lungs and the serum levels of interleukin-6 (IL-6) and tumor necrosis factor- $\alpha$ (TNF- $\alpha$ ) (measured by ELISA), as well as nuclear factor- $\kappa \mathrm{B}$ protein expression in mice with LPS-induced ALI. Moreover, treatment with sulforaphane significantly inhibited prostaglandin E2 (PGE2) production, and cyclooxygenase-2 (COX-2), matrix metalloproteinase-9 (MMP-9) protein expression (as shown by western blot analysis), as well as inducible nitric oxide synthase (iNOS) activity in mice with LPS-induced ALI. Lastly, we noted that pre-treatment with sulforaphane activated the nuclear factorE2-related factor 2 (Nrf2)/antioxidant response element (ARE) pathway in the mice with LPS-induced ALI. These findings demonstrate that sulforaphane exerts protective effects against LPS-induced ALI through the Nrf2/ARE pathway. Thus, sulforaphane may be a potential a candidate for use in the treatment of ALI.
\end{abstract}

Correspondence to: Dr Xixin Yan, Department of Respiratory Medicine, The Second Hospital of Hebei Medical University, 215 Heping West Road, Xinhua, Shijiazhuang, Hebei 050000, P.R. China

E-mail: yan66002952@163.com

Key words: sulforaphane, lipopolysaccharide, acute lung injury, nuclear factor-E2-related factor 2/antioxidant response element

\section{Introduction}

Acute lung injury (ALI) is a disorder of acute lung inflammation and is clinically characterized by the enhanced permeability of the alveolar-capillary barrier and disordered air-exchange function. It is also known as acute respiratory distress syndrome (ARDS) (1). Its typical pathological characteristics include injury to pulmonary capillary endothelial cells and alveolar epithelial cells, extensive pulmonary edema, microatelectasis, microthrombosis and microcirculation disturbance (2). Serious infection, trauma, shock, intoxication and inhalation of toxic gases are the most common causes of ALI (3). ALI/ARDS is a common critical disease with high morbidity and fatality rates (4). With improvements in medical technology and comprehensive treatment methods, the fatality rate has decreased to a certain extent; however, it remains as high as $30-40 \%$ (5). Moreover, the mechanisms responsible for the development of ALI/ARDS are complex and are not yet fully understood $(4,5)$. Currently, clinical therapy is mainly comprehensive, and no specific treatment method is available (6). Consequently, studies on the prevention and treatment of ALI/ARDS are of great importance (4).

ALI is a disease characterized by direct or indirect damage to lung epithelial and endothelial cells (7). The mechanisms responsible for the development of this disease, however, remain to be elucidated (7). It was originally considered that the root cause of ALI is cell activation by pathogenic factors and bodily fluids, which cause inflammatory response syndrome, and pathological processes such as alveolar collapse, an imbalance in the ventilation/perfusion ratio and a decrease in lung compliance. The imbalance between inflammatory reactions and anti-inflammatory reactions is of great significance in the development of ALI $(7,8)$. The disease can be divided into the early and late stages according to its pathological characteristics (9). Acute inflammation of the lung tissue and damage to lung cells are the pathological characteristics of the early stages of the disease (10). Pulmonary fibrosis and lung cell damage are the main characteristics of the later stages of the disease. In recent years, a certain amount of progress has been made as regards the understanding of the mechanisms responsible for ALI (11). The inflammation/anti-inflammatory mechanism, oxidation/antioxidant mechanism, coagulation/fibrinolysis 
system and cell apoptosis mechanism have been identifed to be involved in the development of ALI (12). In addition, in this study, we investigated whether sulforaphane exerts anti-inflammatory effects against lipopolysaccharide (LPS)-induced ALI in mice through the Nrf2/ARE pathway.

Sulforaphane (1-isothiocyanate-4-methyl sulfonyl butane) is also known as 'raphanin'. In terms of its chemical components, these are derivatives of isothiocyanate which is soluble in water with a relative molecular mass of 177.3 and molecular formula as C6H11S2NO (13). Sulforaphane as an agonist of nuclear factor-E2-related factor 2 (Nrf2), is extracted from cruciferous vegetables (such as broccoli, brussels sprouts and cabbage) (14). It has previously been demonstrated that sulforaphane exerts protective effects on multiple organs, such as the liver, lungs, kidneys, heart and nervous system by activating Nrf2 (14). Sulforaphane has also been shown to exert protective effects on mouse peritoneal macrophages positive for Nrf2; however, these effects were attenuated in macrophages negative for Nrf2 and the authors concluded that sulforaphane exerts its anti-inflammatory via the activation of Nrf2 (15). Thus, in the present study, we aimed to investigate the anti-inflammatory effects of sulforaphane and the mechanisms through which it protects against LPS-induced ALI. We also aimed to investigate the signaling pathways involved.

$\mathrm{Nrf} 2$ is an important transcription factor and a key regulator of the activation of antioxidant genes to exogenous stimulations. Reactive oxygen species (ROS) give rise to the disruption of the Nrf2/Kelch-like ECH-associated protein-1 (Keap1) complex and Nrf2 from the cytoplasm into the nucleus where Keap1 dimerizes with antioxidant response element (ARE) DNA sequence ultimately activates the expression of ARE-dependent genes.

\section{Materials and methods}

Materials. The interleukin-6 (IL-6), tumor necrosis factor- $\alpha$ (TNF- $\alpha$ ), prostaglandin E2 (PGE2) and nitric oxide (NO) commercial enzyme-linked immunosorbent assay (ELISA) kits were purchased from the Nanjing Jiancheng Bioengineering Institute, Nanjing, China. The chemical structure of sulforaphane ( $\geq 95 \%$; Sigma-Aldrich Co. LLC, St. Louis, MO, USA) is illustrated in Fig. 1.

Animals. Male BALB/c mice (8-10 weeks old, weighing 18-20 g) were purchased from the Second Hospital of Hebei Medical University (Hebei, China). All experimental procedures were strictly in accordance with the Guide for the Care and Use of Laboratory Animals of The Second Hospital of Hebei Medical University. This study was approved by the Ethics Committee of The Second Hospital of Hebei Medical University. The male $\mathrm{BALB} / \mathrm{c}$ mice were housed in standard environmental conditions of light $\left(12 / 12 \mathrm{~h}\right.$ light/dark cycle), temperature $\left(22 \pm 2^{\circ} \mathrm{C}\right)$ and $50 \pm 10 \%$ humidity, with free access to food and water.

Mouse model of ALI and experimental groups. The BALB/c mice were randomly divided into 3 groups as follows: the control, the model and sulforaphane-treated groups ( $\mathrm{n}=8$ per group). Prior to the induction of ALI, sulforaphane $(50 \mathrm{mg} / \mathrm{kg}$ ) was administered by an intraperitoneal (i.p.) injection, as previously described (16). Seventy-two hours later, $25 \mu \mathrm{g}$ LPS were instilled in $50 \mu \mathrm{l}$ phosphate-buffered saline (PBS) to induce lung injury.

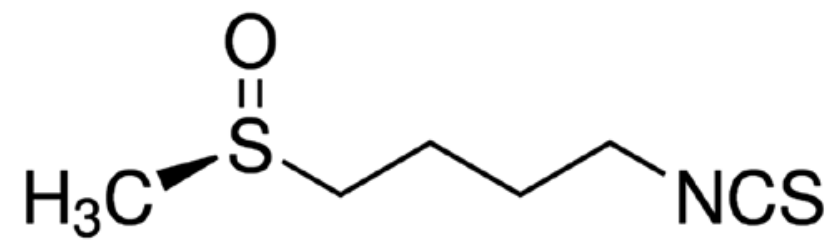

Figure 1. Chemical structure of sulforaphane.

Lactate dehydrogenase ( $L D H$ ) assay. At $12 \mathrm{~h}$ after the LPS challenge, the mice were sacrificed by decollation and lung tissue was acquired and mixed with freshly prepared reaction mixture. The lung samples were incubated with LPS in the dark for $30 \mathrm{~min}$ at room temperature. The absorbance was measured using a microplate reader (BMG Labtech, Ortenberg, Germany) at $490 \mathrm{~nm}$.

Measurement of the wet-to-dry ratio of the lungs. At $12 \mathrm{~h}$ after the LPS challenge, the mice were sacrificed by decollation and lung tissue was acquired and the right lungs were excised and weighed. Subsequently, the right lungs were placed in an incubator at $80^{\circ} \mathrm{C}$ for $48 \mathrm{~h}$ and weighed. The wet-to-dry ratio of the lungs was calculated as follows: dry weight/wet weight $\mathrm{x} 100$.

ELISA. At $12 \mathrm{~h}$ after the LPS challenge, lung samples were acquired and lavaged with $500 \mu 1$ sterile PBS. Lung samples were centrifuged at $3,000 \mathrm{rpm}$ for $10 \mathrm{~min}$ at $4^{\circ} \mathrm{C}$. The IL-6, TNF- $\alpha$ and PGE2 activities were analyzed using a microplate reader (BMG Labtech), in accordance with the manufacturer's instructions (Nanjing Jiancheng Bioengineering Institute).

Western blot analysis for nuclear factor $-\kappa B(N F-\kappa B)$, cyclooxygenase-2 (COX-2) and matrix metalloproteinase-9 (MMP-9) expression. At $12 \mathrm{~h}$ after the LPS challenge, lung samples were acquired and lavaged with $500 \mu 1$ sterile PBS. The lung samples were homogenized with RIPA buffer (Beyotime Biotech, Shanghai, China) and centrifuged at 3,000 rpm for $10 \mathrm{~min}$ at $4^{\circ} \mathrm{C}$. The protein concentration was determined with the BCA protein assay (Beyotime Biotech). The homogenized samples were separated by $10 \%$ sodium dodecyl sulfate-polyacrylamide gel electrophoresis (SDS-PAGE) and electrophoretically transferred onto nitrocellulose membranes (Millipore, Billerica, MA, USA), which were blocked with $5 \%$ non-fat milk with Trisbuffered saline for $2 \mathrm{~h}$ at room temperature. The membranes were incubated overnight at $4{ }^{\circ} \mathrm{C}$ with primary antibodies to NF- $\mathrm{B}$ (sc-109), COX-2 (sc-514489) and MMP-9 (sc-21733; all from Santa Cruz Biotechnology, Inc., Santa Cruz, CA, USA) overnight at $4{ }^{\circ} \mathrm{C}$ followed by incubation with horseradish peroxidase-conjugated secondary antibody (sc-69916; Santa Cruz Biotechnology, Inc.) for $2 \mathrm{~h}$ at room temperature. All blots were washed with enhanced chemiluminescence (ECL) reagents according to the manufacturer's instructions and analyzed using Bio-Rad Quantity One software (Bio-Rad, Hercules, CA, USA).

RNA isolation and quantitative PCR (qPCR). At $12 \mathrm{~h}$ after the LPS challenge, lung samples were acquired and were used in the extraction of total RNA using TRIzol reagent (Invitrogen, Carlsbad, CA, USA). Subsequently, the RNA was purified using the RNeasy mini kit according to the manufacturer's 


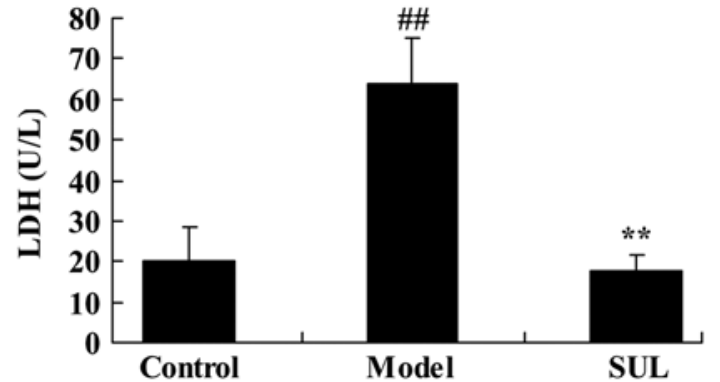

Figure 2. Anti-inflammatory effects of sulforaphane on lactate dehydrogenase (LDH) activity in mice with lipopolysaccharide-induced acute lung injury (ALI). Control, control (untreated) group; model, mouse model of lipopolysaccharide-induced ALI; SUL, sulforaphane-treated group. ${ }^{\# /} \mathrm{P}<0.01$ compared with the control group; ${ }^{* *} \mathrm{P}<0.01$ compared with the model group.

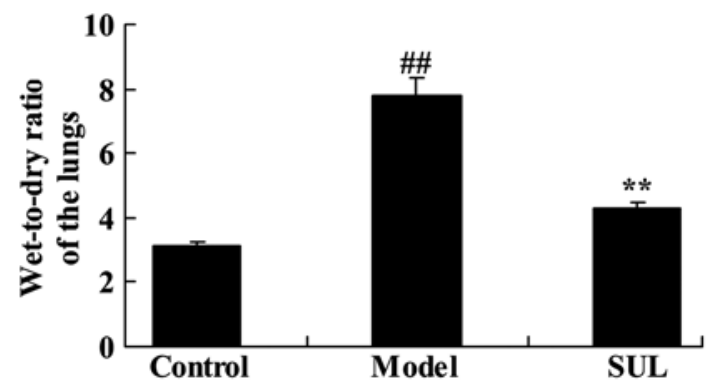

Figure 3. Anti-inflammatory effects of sulforaphane on wet-to-dry ratio of the lungs of mice with lipopolysaccharide-induced acute lung injury (ALI). Control, control (untreated) group; model, mouse model of lipopolysaccharide-induced ALI; SUL, sulforaphane-treated group. ${ }^{\# \#} \mathrm{P}<0.01$ compared with the control group; ${ }^{* *} \mathrm{P}<0.01$ compared with the model group.

instructions (Qiagen, Valencia, CA, USA). cDNA was synthesized using an iScript cDNA synthesis kit (Bio-Rad). The 7000 Sequence Detection system (Applied Biosystems, Foster City, CA, USA) was used to perform qPCR. The sequences of the primers used for gene amplification were as follows: Nrf2 forward, 5'-CCCACAAGTTCGGCATCCAC-3' and reverse, 5'-TGGCGATTCCTCTGGCGTCT-3'; and $\beta$-actin forward, 5'-CGCGACATCAAGGAGAAGCTG-3' and reverse, 5'-ATTGCCAATGGGTGATACCTG-3'.

Measurement of NO production. At $12 \mathrm{~h}$ after the LPS challenge, lung samples were acquired and lavaged with $500 \mu 1$ sterile PBS. The lung samples were homogenized with RIPA buffer (Beyotime Biotech) and centrifuged at 3,000 rpm for $10 \mathrm{~min}$ at $4^{\circ} \mathrm{C}$. The supernatant was incubated using a microplate reader (BMG Labtech), for $10 \mathrm{~min}$ at room temperature and the optical density was measured at $540 \mathrm{~nm}$.

Statistical analysis. Data are expressed as the means $\pm \mathrm{SD}$, and were analyzed by one-way analysis of variance (ANOVA); variations between the different groups were compared using the Tukey-Kramer post hoc test. A P-value $<0.05$ was deemed asto indicate a statistically significant difference.

\section{Results}

Anti-inflammatory effects of sulforaphane on LDH activity in mice with LPS-induced ALI. To evaluate the effects of sulfora-
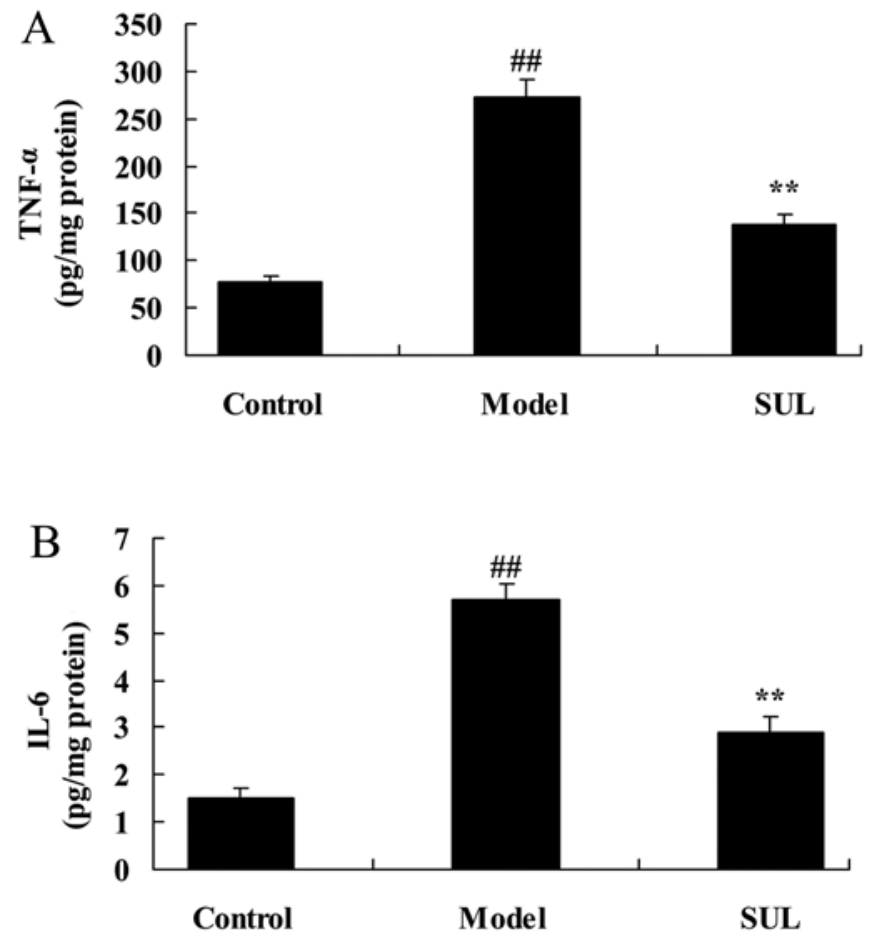

Figure 4. Anti-inflammatory effects of sulforaphane on the lungs of mice with lipopolysaccharide-induced acute lung injury (ALI). Anti-inflammatory effects of sulforaphane on interleukin (IL)- 6 and tumor necrosis factor- $\alpha$ (TNF- $\alpha$ ) activities in lungs of mice with lipopolysaccharide-induced ALI. Control, control (untreated) group; model, mouse model of lipopolysaccharide-induced ALI; SUL, sulforaphane-treated group. ${ }^{\# \#} \mathrm{P}<0.01$ compared with the control group; ${ }^{* *} \mathrm{P}<0.01$ compared with the model group.

phane on LDH activity in mice with LPS-induced ALI, we established a model of LPS-induced ALI and measured LDH activity. LPS effectively induced LDH activity in the mice compared to the untreated control group (Fig. 2). Treatment with sulforaphane effectively inhibited LDH activity in the mice with ALI (Fig. 2).

Anti-inflammatory effects of sulforaphane on the wet-todry ratio of the lungs of mice with LPS-induced ALI. We subsequently determined the anti-inflammatory effects of sulforaphane on the wet-to-dry ratio of the lungs of mice with LPS-induced ALI. LPS-induced ALI notably increased the wet-to-dry ratio of the lungs of mice with ALI, compared to lungs from the mice in the control group (Fig. 3). We also noted that treatment with sulforaphane decreased the wet-to-dry ratio, almost to the same level as the control group. These data demonstrated that pre-treatment with sulforaphane markedly reduced the wet-to-dry ratio of lungs of mice with ALI.

Anti-inflammatory effects of sulforaphane on the lungs of mice with LPS-induced ALI. To examine the anti-inflammatory effects of sulforaphane on the lungs of mice with LPS-induced ALI, the activities of IL- 6 and TNF- $\alpha$ were surveyed following the induction of ALI by LPS. The IL- 6 and TNF- $\alpha$ activities were markedly increased in the mice with LPS-induced ALI, compared to the mice in the untreated control group (Fig. 4). Treatment with sulforaphane led to a significant decrease in IL-6 and TNF- $\alpha$ activities in the mice with LPS-induced ALI (Fig. 4). 
A

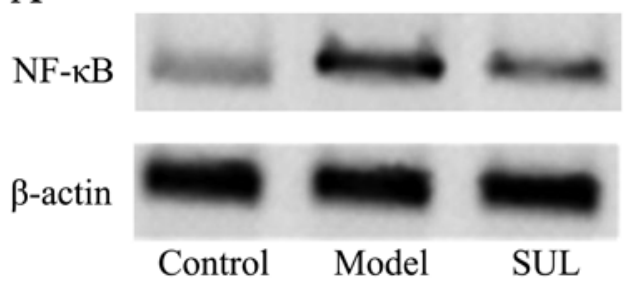

B

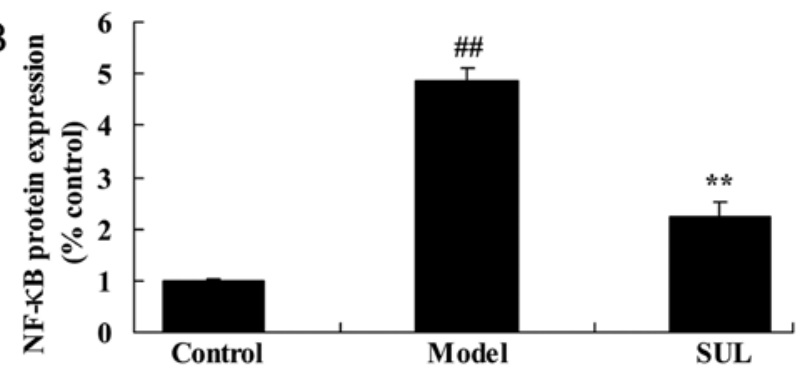

Figure 5. Anti-inflammatory effects of sulforaphane on nuclear factor- $\kappa \mathrm{B}(\mathrm{NF}-\kappa \mathrm{B})$ protein expression in the lungs of mice with lipopolysaccharide-induced acute lung injury (ALI). Anti-inflammatory effects of sulforaphane on NF- $\mathrm{B}$ protein expression in the lungs as measured by (A) western blot analysi and (B) statistical analysis of NF- $\mathrm{B}$ protein expression in lungs of mice with lipopolysaccharide-induced ALI. Control, control (untreated) group; model, mouse model of lipopolysaccharide-induced ALI; SUL, sulforaphane-treated group. ${ }^{\# \#} \mathrm{P}<0.01$ compared with the control group; ${ }^{* *} \mathrm{P}<0.01$ compared with the model group.

A

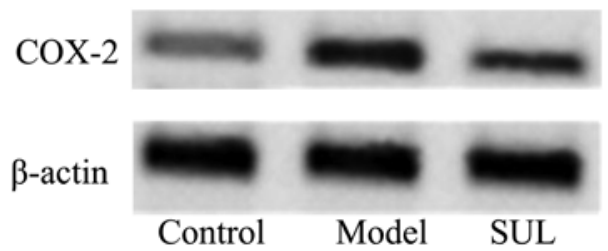

B

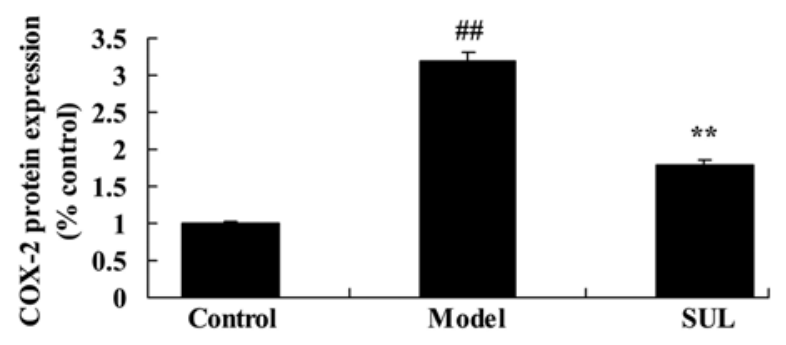

Figure 6. Anti-inflammatory effects of sulforaphane on COX-2 protein expression in the lungs of mice with lipopolysaccharide-induced acute lung injury (ALI). Anti-inflammatory effects of sulforaphane on COX-2 protein expression in the lungs as measured by (A) western blot analysis and (B) statistical analysis of COX-2 protein expression in lungs of mice with lipopolysaccharide-induced ALI. Control, control (untrteated) group; model, mouse model of lipopolysaccharide-induced ALI; SUL, sulforaphane-treated group. ${ }^{\# \#} \mathrm{P}<0.01$ compared with the control group; ${ }^{* *} \mathrm{P}<0.01$ compared with the model group.

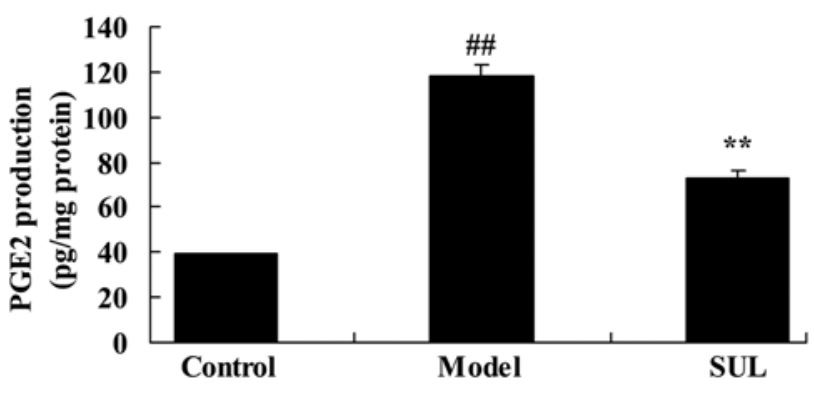

Figure 7. Anti-inflammatory effects of sulforaphane on prostaglandin E2 (PGE2) production in the lungs of mice with lipopolysaccharide-induced acute lung injury (ALI). Control, control (untreated) group; model, mouse model of lipopolysaccharide-induced ALI; SUL, sulforaphane-treated group. ${ }^{\# \#} \mathrm{P}<0.01$ compared with the control group; ${ }^{* * *} \mathrm{P}<0.01$ compared with the model group.

Anti-inflammatory effects of sulforaphane on $N F-\kappa B$ protein expression in lungs of mice with LPS-induced ALI. To examine the anti-inflammatory mechanisms of action of sulforaphane on $N F-\kappa B$ protein expression in the lungs of mice with LPS-induced ALI, western blot analysis was used to measure the $\mathrm{NF}-\kappa \mathrm{B}$ protein expression levels. The results revealed that $\mathrm{NF}-\kappa \mathrm{B}$ protein expression was higher in the lungs of mice with LPS-induced ALI, compared to the lungs of mice in the control group (Fig. 5). The sulforaphane-treated mice exhibited a significant decrease in NF- $\kappa \mathrm{B}$ protein expression in the lungs compared to the mice with LPS-induced ALI (Fig. 5).

Anti-inflammatory effects of sulforaphane on $C O X-2$ protein expression in the lungs of mice with LPS-induced ALI. As shown in Fig. 6, there was a significant increase in COX-2 protein expression in the lungs of mice with LPS-induced ALI, compared to the mice in the control group. However, treatment with sulforaphane significantly suppressed COX-2 protein expression in the lungs of mice with LPS-induced ALI (Fig. 6).

Anti-inflammatory effects of sulforaphane on PGE2 production in the lungs of mice with LPS-induced ALI. PGE2 production in the lungs of mice with LPS-induced ALI was significantly higher than that in the lungs of mice in the control group (Fig. 7). We noted that PGE2 production was significantly reduced by treatment with sulforaphane (Fig. 7).

Anti-inflammatory effects of sulforaphane on MMP-9 protein expression in the lungs of mice with LPS-induced ALI. LPS markedly increased MMP-9 protein expression in the lungs of mice with LPS-induced ALI. Western blot analysis was used to measure the MMP-9 protein expression levels. MMP-9 protein expression was markedly increased in the mice with LPS-induced ALI, compared to the mice in the control group (Fig. 8). In the lungs of mice treated with sulforaphane, we noted a statistically significant decrease in MMP-9 protein expression (Fig. 8).

Anti-inflammatory effects of sulforaphane on NO production in the lungs of mice with LPS-induced ALI. There was a significant increase in the NO levels in the lungs of mice with LPS-induced ALI, compared to the mice in the control group (Fig. 9). However, treatment with sulforaphane significantly decreased the NO levels (Fig. 9). 
A

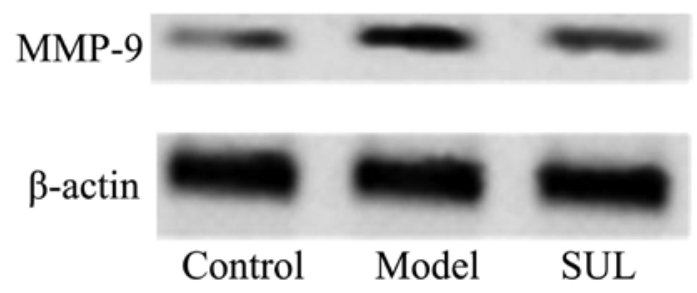

$\mathrm{B}$

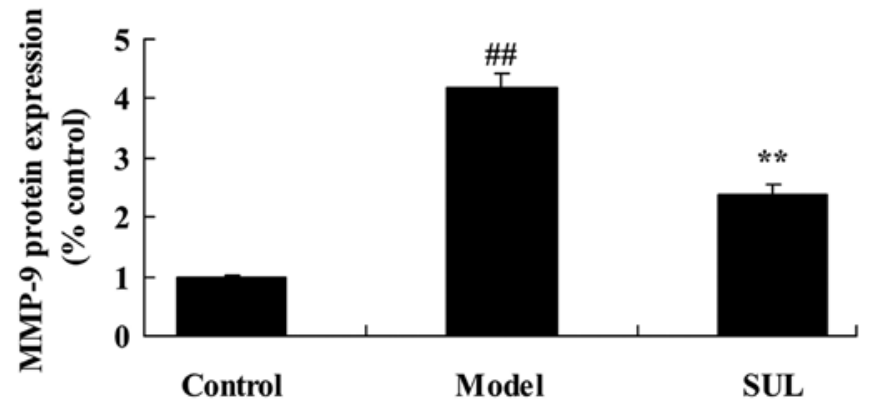

Figure 8. Anti-inflammatory effects of sulforaphane on matrix metalloproteinase-9(MMP-9) protein expression of the lungs of mice with lipopolysaccharide-induced acute lung injury (ALI). Anti-inflammatory effects of sulforaphane on MMP-9 protein expression in the lungs, measured by (A) western blot analysis (A) and (B) statistical analysis of MMP-9 protein expression in mice with lipopolysaccharide-induced ALI. Control, control (untreated) group; model, mouse model of lipopolysaccharide-induced ALI; SUL, sulforaphane-treated group. ${ }^{\# \#} \mathrm{P}<0.01$ compared with the control group; ${ }^{* *} \mathrm{P}<0.01$ compared with the model group.

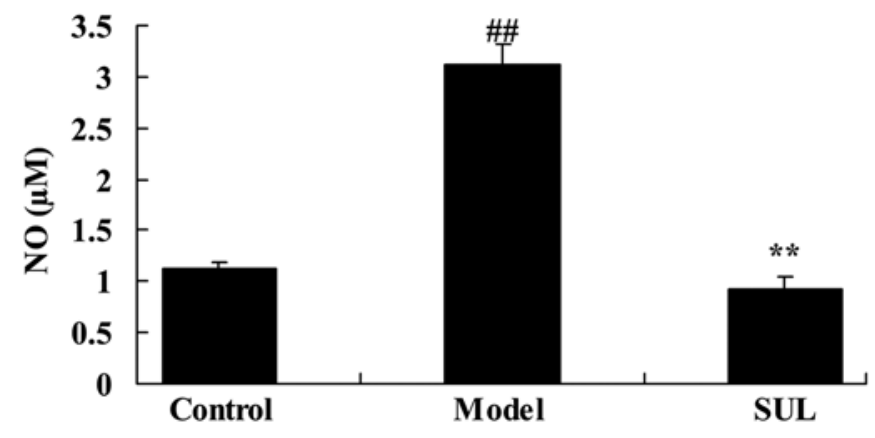

Figure 9. Anti-inflammatory effects of sulforaphane on nitric oxide (NO) in the lungs of mice with lipopolysaccharide-induced acute lung injury (ALI) Control, control (untreated) group; model, mouse model of lipopolysaccharide-induced ALI; SUL, sulforaphane-treated group. ${ }^{\# \#} \mathrm{P}<0.01$ compared with the control group; ${ }^{* *} \mathrm{P}<0.01$ compared with the model group.

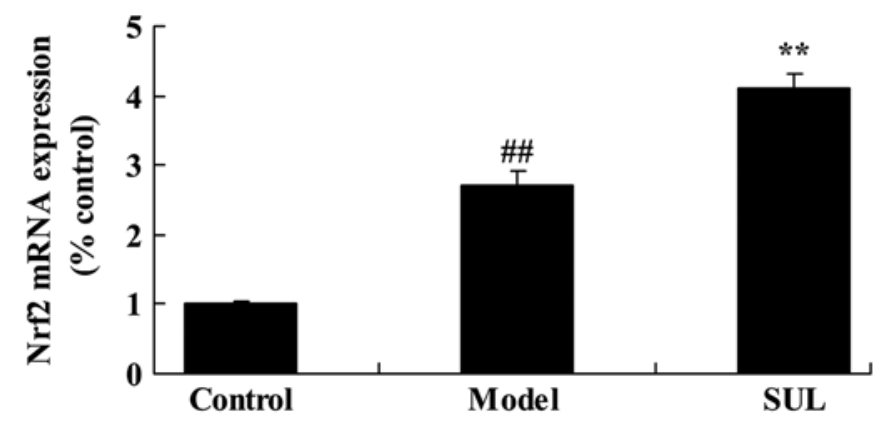

Figure 10. Anti-inflammatory effects of sulforaphane on nuclear factorE2-related factor 2 ( Nrf2) mRNA expression in the lungs of mice with lipopolysaccharide-induced acute lung injury (ALI). Control, control (untreated) group; model, mouse model of lipopolysaccharide-induced ALI; SUL, sulforaphane-treated group. ${ }^{\# \#} \mathrm{P}<0.01$ compared with the control group; ${ }^{* *} \mathrm{P}<0.01$ compared with the model group

Anti-inflammatory effects of sulforaphane on Nrf $2 \mathrm{mRNA}$ expression in the lungs of mice with LPS-induced ALI. Compared to the untreated control group, Nrf2 mRNA expression in the lungs of mice with LPS-induced ALI was significantly increased (Fig. 10). Treatment with sulforaphane led to a further significant increase in Nrf2 mRNA expression compared to the mice with LPS-induced ALI not treated with sulforaphane (Fig. 10).

\section{Discussion}

ALI is a disorder of lung inflammation and is clinically characterized by the enhanced permeability of the alveolarcapillary barrier and disordered air-exchange function (17). Its typical pathological characteristics include injury to pulmonary capillary endothelial cells and alveolar epithelial cells, extensive pulmonary edema, microatelectasis, microthrombosis and microcirculation disturbance (2). It commonly occurs following serious infection, trauma, shock, intoxication and inhalation of toxic gases. ALI (also known as ARDS) is a common critical disease with a high mortality rate (4). The mechanisms repsonsible for the development of ALI are quite complex and have not yet been fully elucidated. Consequently, it is still an important topic which requires further research in today's medical industry. In the present study, we demonstrated that sulforaphane significantly decreased LDH activity and the wet-to-dry ratio of the lungs of mice with LPS-induced ALI.

Excessive lung inflammation response is a typical pathological characteristic of ALI/ARDS (10). Its pathological characteristics are widespread and are very difficult to control, leading to a cascade reaction (12). According to different pathological stages of inflammation, it can be divided into the inflammatory exudation phase in the early stages and fiber hyperplasia in the later stages of the disease (18). The administration of anti-inflammatories constitutes a therapy specific to inflammatory reactions, and such treatment can inhibit inflammatory reactions, reduce damage and is an effective method of treating lung injury during the early stages (19). The findings of our study revealed that treatment with sulforaphane significantly decreased the IL- 6 and TNF- $\alpha$ activities in mice with LPS-induced ALI. In a previous study, it was demonstrated that sulforaphane inhibited the LPS-stimulated inflammatory response by modulating $\mathrm{NF}-\kappa \mathrm{B}$ in human monocytes (20). Nallasamy et al indicated that sulforaphane reduced vascular inflammation by interfering with the $N F-\kappa B$ pathway in mice (21).

$\mathrm{NF}-\kappa \mathrm{B}$ is a nuclear factor present in the majority of cells. It has a very important effect on the growth, differentiation, adhesion, apoptosis and inflammation of cells $(22,23)$. While in a state of inactivity, it combines with IKB in the cytoplasm in an inactive state. In cases of external stimulation, IKB undergoes rapid phosphorylation degradation so as to activate 
$\mathrm{NF}-\kappa \mathrm{B}$ and begin nuclear translocation (11). After NF- $\mathrm{NB}$ is activated, it inevitably regulates the gene expression of inflammatory cytokines participating in the inflammatory reaction and mediates the development of ALI $(24,11)$. Epoxidase is an essential rate-limiting enzyme which is involved in the process of prostaglandin synthesis (25). It has two isozymes, COX-1 and COX-2. COX-2 is an instant and early gene. Its high expression is induced by a variety of genes (26). The activation of $\mathrm{NF}-\kappa \mathrm{B}$ induces the activation of $\mathrm{COX}-2$, which may increase the levels of the endogenous immune inhibitor, PGE2, which causes immunosuppression following infection, thus increasing the occurrence rate of lung injury and the fatality rate (27). In the present study, sulforaphane significantly suppressed NF- $\mathrm{BB}$ and COX-2 protein expression in mice with LPS-induced ALI. Nallasamy et al indicated that sulforaphane reduced vascular inflammation by interfering with the $\mathrm{NF}-\kappa \mathrm{B}$ pathway in mice (21). Shan et al suggested that sulforaphane downregulates $\mathrm{COX}-2$ expression by inhibiting $\mathrm{NF}-\kappa \mathrm{B}$ in human bladder T24 cells (28).

PGE2 is a prostaglandin which plays a complex biological role in the ALI/ARDS inflammatory reaction process (29). PGE2 synthase is the key enzyme for the last step of PGE2 synthesis. Of the detected three types of PGE2 synthase, microsomal prostaglandin $\mathrm{E}_{2}$ synthase-1 (mPGES-1), as the only induced enzyme, has been noted to be upregulated in response to various types of inflammation (29). In ventilator-associated lung injury in mice, mPGES-1-derived PGE2 has been shown to play a role in the onset of lung injury and pulmonary edema, and can promote pathological reactions in a variety of effector cells and can activate cytokines and inflammatory reactions $(30,31)$. In the present study, we demonstrated that treatment with sulforaphane significantly decreased PGE2 production in mice with LPS-induced ALI. Choi et al previously suggested that sulforaphane inhibits IL-1 $\beta$-induced proliferation by decreasing the production of MMP, COX-2 and PGE2 (32).

It has previously been suggested that ALI may trigger the activation of a variety of effector cells, inflammatory reactions and cytokines (33). MMPs are a family of genetically related endopeptidases whose activity is zinc-dependent (33). MMP-9 is an important member of the family (34). It has high hydrolysis specificity for type IV and V collagen protein and gelatin (33). It plays a certain regulatory role in matrix reconstruction. The endogenous inhibitor of MMP-9 is tissue matrix metalloproteinase inhibitor-1 (TIMP-1) $(34,35)$. Under physiological conditions, the activity of MMP-9 is inhibited by TIMP-1 (35). Both are under an equilibrium state (35). If an imbalance exists between the two factors participating in airway remodeling, this results in the development of ALI. In the present study, we found that sulforaphane significantly decreased MMP-9 protein expression in mice with LPS-induced ALI. Mao et al demonstrated that sulforaphane attenuates MMP-9 expression following spinal cord injury in mice (36).

NO has a wide range of biological functions, and affects various pathological and physiological processes (37). Endogenous NO in pulmonary circulation can attenuate hypoxic pulmonary vasoconstriction, increase anoxic lung alveolar fluid filling and protect against hypoxia, thus preventing ALI (38). NO inhibits neutrophil migration to the endothelium, thus reducing the generation of inflammatory cytokines (37). NO also inhibits the activation of neutrophile granulocytes and decreases the release of toxic oxidation products, which is conducive to maintaining the integrity of the alveolar-capillary membrane and repairing damage caused by ALI (39). In this study, treatment with sulforaphane significantly decreased the NO levels in the lungs of mice with LPS-induced ALI. Brandenburg et al demonstrated that sulforaphane suppressed LPS-induced inflammation by decreasing NO levels in rat primary microglia (40).

Nrf 2 is an important regulator of cell oxidation. It can regulate the expression of peroxiredoxins, generation II metabolic enzymes, enhance the ability of cells to remove ROS, maintain the oxidation-reduction equilibrium status in cells, and reduce oxidative damage through interaction with ARE (41). Nrf2/ARE is an edogenous antioxidant stress pathway (42). It plays an important role in the treatment of a variety of diseases (42). It has been demonstrated that Nrf2 is a promising target for the treatment of ALI (41). In the present study, sulforaphane increased Nrf2 gene expression in the lungs of mice with LPS-induced ALI. Chi et al demonstrated that sulforaphane reduced apoptosis and protected against liver injury-induced ischemic reperfusion by activating the Nrf2/ARE pathway (14). Lin et al revealed that sulforaphane suppressed LPS-induced inflammation through the Nrf2 pathway in mouse peritoneal macrophages (15).

In conclusion, the findings of this study demonstrate that sulforaphane protects against LPS-induced ALI in mice by exerting anti-inflammatory effects, by mediating the production of NF- $\kappa \mathrm{B}, \mathrm{COX}-2, \mathrm{PGE} 2$ and $\mathrm{NO}$ and upregulating Nrf2 expression. Moreover, the present findings provide a scientific foundation for the use of sulforaphane as a prophylactic treatment for ALI in clinical settings.

\section{References}

1. Emr BM, Roy S, Kollisch-Singule M, Gatto LA, Barravecchia M, Lin X, Young JL, Wang G, Liu J, Satalin J, et al: Electroporationmediated gene delivery of $\mathrm{Na}^{+}, \mathrm{K}^{+}$-ATPase, and $\mathrm{ENaC}$ subunits to the lung attenuates acute respiratory distress syndrome in a two-hit porcine model. Shock 43: 16-23, 2015.

2. Wang L, Taneja R, Wang W, Yao LJ, Veldhuizen RA, Gill SE, Fortin D, Inculet R, Malthaner R and Mehta S: Human alveolar epithelial cells attenuate pulmonary microvascular endothelial cell permeability under septic conditions. PLoS One 8: e55311, 2013.

3. Ather JL, Alcorn JF, Brown AL, Guala AS, Suratt BT, JanssenHeininger YM and Poynter ME: Distinct functions of airway epithelial nuclear factor-kappaB activity regulate nitrogen dioxide-induced acute lung injury. Am J Respir Cell Mol Biol 43: 443-451, 2010.

4. Fard N, Saffari A, Emami G, Hofer S, Kauczor HU and Mehrabi A: Acute respiratory distress syndrome induction by pulmonary ischemia-reperfusion injury in large animal models. J Surg Res 189: 274-284, 2014.

5. Yang B, Huang W, Han J and Liang Z: Study of the role of epidermal growth factor on lung fluid transport in rabbits with acute lung injury caused by endotoxin. Exp Ther Med 4: 611-614, 2012.

6. Yan YM, Li YD, Song XL, Liu M, Diao F, Wang Y, Sun Y, Wang $\mathrm{ZH}$ and $\mathrm{Lu} \mathrm{J}$ : Therapeutic effects of inhaling aerosolized surfactant alone or with dexamethasone generated by a novel noninvasive apparatus on acute lung injury in rats. J Trauma Acute Care Surg 73: 1114-1120, 2012.

7. Bhandary YP, Velusamy T, Shetty P, Shetty RS, Idell S, Cines DB, Jain D, Bdeir K, Abraham E, Tsuruta Y, et al: Post-transcriptional regulation of urokinase-type plasminogen activator receptor expression in lipopolysaccharide-induced acute lung injury. Am J Respir Crit Care Med 179: 288-298, 2009.

8. Wang X, Zhang L, Duan W, Liu B, Gong P, Ding Y and Wu X: Anti-inflammatory effects of triptolide by inhibiting the NF- $\kappa \mathrm{B}$ signalling pathway in LPS-induced acute lung injury in a murine model. Mol Med Rep 10: 447-452, 2014. 
9. Schmidt AE and Adamski J; Education Committee of the Academy of Clinical Laboratory Physicians and Scientists: Pathology consultation on transfusion-related acute lung injury (TRALI). Am J Clin Pathol 138: 498-503, 2012.

10. Wang ZY, Wu SN, Zhu ZZ, Yang BX and Zhu X: Inhaled unfractionated heparin improves abnormalities of alveolar coagulation, fibrinolysis and inflammation in endotoxemia-induced lung injury rats. Chin Med J (Engl) 126: 318-324, 2013.

11. Zhang JZ, Liu Z, Liu J, Ren JX and Sun TS: Mitochondrial DNA induces inflammation and increases TLR9/NF- $\kappa \mathrm{B}$ expression in lung tissue. Int J Mol Med 33: 817-824, 2014.

12. Irwin DC, Baek JH, Hassell K, Nuss R, Eigenberger P, Lisk C, Loomis Z, Maltzahn J, Stenmark KR, Nozik-Grayck E and Shetty S: Hemoglobin-induced lung vascular oxidation, inflammation, and remodeling contribute to the progression of hypoxic pulmonary hypertension and is attenuated in rats with repeated-dose haptoglobin administration. Free Radic Biol Med 82: 50-62, 2015.

13. Dominguez-Perles R, Medina S, Moreno DA, Garcia-Viguera C Ferreres $\mathrm{F}$ and Gil-Izquierdo A: A new ultra-rapid UHPLC MS/MS method for assessing glucoraphanin and sulforaphane bioavailability in human urine. Food Chem 143: 132-138, 2014.

14. Chi X, Zhang R, Shen N, Jin Y, Alina A, Yang S and Lin S: Sulforaphane reduces apoptosis and oncosis along with protecting liver injury-induced ischemic reperfusion by activating the Nrf2/ARE pathway. Hepatol Int 9: 321-329, 2015.

15. Lin W, Wu RT, Wu T, Khor TO, Wang H and Kong AN: Sulforaphane suppressed LPS-induced inflammation in mouse peritoneal macrophages through Nrf2 dependent pathway. Biochem Pharmacol 76: 967-973, 2008

16. Benedict AL, Mountney A, Hurtado A, Bryan KE, Schnaar RL, Dinkova-Kostova AT and Talalay P: Neuroprotective effects of sulforaphane after contusive spinal cord injury. J Neurotrauma 29: 2576-2586, 2012

17. Wang CT, Zhang L, Wu HW, Wei L, Xu B and Li DM: Doxycycline attenuates acute lung injury following cardiopulmonary bypass: Involvement of matrix metalloproteinases. Int J Clin Exp Pathol 7: 7460-7468, 2014.

18. Chevalier S, Cury FL, Scarlata E, El-Zayat E, Hamel L, Rocha J, Zouanat FZ, Moussa S, Scherz A, Elhilali M and Anidjar M: Endoscopic vascular targeted photodynamic therapy with the photosensitizer WST11 for benign prostatic hyperplasia in the preclinical dog model. J Urol 190: 1946-1953, 2013.

19. Joo Choi R, Cheng MS and Shik Kim Y: Desoxyrhapontigenin up-regulates Nrf2-mediated heme oxygenase-1 expression in macrophages and inflammatory lung injury. Redox Biol 2 . 504-512, 2014

20. Reddy SA, Shelar SB, Dang TM, Lee BN, Yang H, Ong SM, $\mathrm{Ng}$ HL, Chui WK, Wong SC and Chew EH: Sulforaphane and its methylcarbonyl analogs inhibit the LPS-stimulated inflammatory response in human monocytes through modulating cytokine production, suppressing chemotactic migration and phagocytosis in a NF- $\kappa$ B- and MAPK-dependent manner. Int Immunopharmacol 24: 440-450, 2015.

21. Nallasamy P, Si H, Babu PV, Pan D, Fu Y, Brooke EA, Shah H, Zhen W, Zhu H, Liu D, et al: Sulforaphane reduces vascular inflammation in mice and prevents TNF- $\alpha$-induced monocyte adhesion to primary endothelial cells through interfering with the NF- $\kappa$ B pathway. J Nutr Biochem 25: 824-833, 2014.

22. Arslan S, Korkmaz Ö, Özbilüm N and Berkan Ö: Association between NF- $\kappa \mathrm{BI}$ and NF- $\kappa \mathrm{BIA}$ polymorphisms and coronary artery disease. Biomed Rep 3: 736-740, 2015.

23. Mankan AK, Lawless MW, Gray SG, Kelleher D and McManus R: NF-kappaB regulation: the nuclear response. J Cell Mol Med 13: 631-643, 2009

24. Liang D, Sun Y, Shen Y, Li F, Song X, Zhou E, Zhao F, Liu Z, $\mathrm{Fu}$ Y, Guo M, et al: Shikonin exerts anti-inflammatory effects in a murine model of lipopolysaccharide-induced acute lung injury by inhibiting the nuclear factor-kappaB signaling pathway. Int Immunopharmacol 16: 475-480, 2013

25. Trapani L, Segatto M, Ascenzi P and Pallottini V: Potential role of nonstatin cholesterol lowering agents. IUBMB Life 63 : 964-971, 2011
26. Ethridge RT, Chung DH, Slogoff M, Ehlers RA, Hellmich MR, Rajaraman S, Saito H, Uchida T and Evers BM: Cyclooxygenase-2 gene disruption attenuates the severity of acute pancreatitis and pancreatitis-associated lung injury. Gastroenterology 123: 1311-1322, 2002

27. Santos LA, Ribeiro EL, Barbosa KP, Fragoso IT, Gomes FO, Donato MA, Silva BS, Silva AK, Rocha SW, França ME, et al: Diethylcarbamazine inhibits $\mathrm{NF}-\kappa \mathrm{B}$ activation in acute lung injury induced by carrageenan in mice. Int Immunopharmacol 23 : 153-162, 2014.

28. Shan Y, Wu K, Wang W, Wang S, Lin N, Zhao R, Cassidy A and Bao Y: Sulforaphane down-regulates COX-2 expression by activating p38 and inhibiting NF-kappaB-DNA-binding activity in human bladder T24 cells. Int J Oncol 34: 1129-1134, 2009.

29. Grantham CJ, Izumi T, Lewis DH and Bakhle YS: Effects of endotoxin-induced lung injury on the pharmacokinetics of prostaglandin $E_{2}$ and adenosine in rat isolated lung. Circ Shock 26: 157-167, 1988.

30. Sun Y, Jia Z, Liu G, Zhou L, Liu M, Yang B and Yang T: PPAR agonist rosiglitazone suppresses renal mPGES-1/PGE2 pathway in db/db Mice. PPAR Res 2013: 612971, 2013.

31. Kono K, Toda S, Hora K and Kiyosawa K: Direct hemoperfusion with a beta2-microglobulin-selective adsorbent column eliminates inflammatory cytokines and improves pulmonary oxygenation. Ther Apher Dial 13: 27-33, 2009.

32. Choi YJ, Lee WS, Lee EG, Sung MS and Yoo WH: Sulforaphane inhibits IL- $1 \beta$-induced proliferation of rheumatoid arthritis synovial fibroblasts and the production of MMPs, COX-2, and PGE2. Inflammation 37: 1496-1503, 2014

33. Menezes LG, Uzuelli JA, Tefé-Silva C, Ramos SG, Santos JE and Martinez JA: Acute lung injury induced by the intravenous administration of cigarette smoke extract. J Bras Pneumol 39: 39-47, 2013.

34. Qi B, Chen HL, Shang D, Dong Y, Zhang GX and Yu L: Effects of hypoxia-inducible factor-1 $\alpha$ and matrix metalloproteinase-9 on alveolar-capillary barrier disruption and lung edema in rat models of severe acute pancreatitis-associated lung injury. Exp Ther Med 8: 899-906, 2014.

35. Kim KH, Burkhart K, Chen P, Frevert CW, Randolph-Habecker J, Hackman RC, Soloway PD and Madtes DK: Tissue inhibitor of metalloproteinase-1 deficiency amplifies acute lung injury in bleomycin-exposed mice. Am J Respir Cell Mol Biol 33: 271-279, 2005.

36. Mao L, Wang HD, Wang XL, Qiao L and Yin HX: Sulforaphane attenuates matrix metalloproteinase-9 expression following spinal cord injury in mice. Ann Clin Lab Sci 40: 354-360, 2010.

37. Liu H, Liang X, Wang D, Zhang H, Liu L, Chen H, Li Y, Duan Q and Xie K: Combination therapy with nitric oxide and molecular hydrogen in a murine model of acute lung injury. Shock 43: 504-511, 2015.

38. Xu D, Niu W, Luo Y, Zhang B, Liu M, Dong H, Liu Y and Li Z: Endogenous estrogen attenuates hypoxia-induced pulmonary hypertension by inhibiting pulmonary arterial vasoconstriction and pulmonary arterial smooth muscle cells proliferation. Int J Med Sci 10: 771-781, 2013.

39. Lin HJ, Wang CT, Niu KC, Gao C, Li Z, Lin MT and Chang CP: Hypobaric hypoxia preconditioning attenuates acute lung injury during high-altitude exposure in rats via up-regulating heat-shock protein 70. Clin Sci (Lond) 121: 223-231, 2011.

40. Brandenburg LO, Kipp M, Lucius R, Pufe T and Wruck CJ Sulforaphane suppresses LPS-induced inflammation in primary rat microglia. Inflamm Res 59: 443-450, 2010.

41. Shan Y, Akram A, Amatullah H, Zhou DY, Gali PL, Maron-Gutierrez T, González-López A, Zhou L, Rocco PR, Hwang D, et al: ATF3 protects pulmonary resident cells from acute and ventilator-induced lung injury by preventing Nrf2 degradation. Antioxid Redox Signal 22: 651-668, 2015.

42. Yu JB, Shi J, Gong LR, Dong SA, Xu Y, Zhang Y, Cao XS and Wu LL: Role of Nrf2/ARE pathway in protective effect of electroacupuncture against endotoxic shock-induced acute lung injury in rabbits. PLoS One 9: e104924, 2014. 